\title{
Risk factors associated with acute in-hospital delirium for patients diagnosed with a hip fracture in the emergency department
}

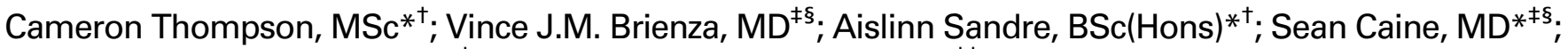 \\ Bjug Borgundvaag, MD, PhD**; Shelley McLeod, $\mathrm{PhD}(\mathrm{c}), \mathrm{MSc}^{*^{\dagger \ddagger}}$
}

\begin{abstract}
CLINICIAN'S CAPSULE
What is known about the topic?

Delirium, or acute confusion, occurs in up to $62 \%$ of patients who have experienced a hip fracture.

What did this study ask?

What risk factors are associated with acute in-hospital delirium for ED patients diagnosed with a hip fracture? What did this study find?

History of neurodegenerative disease or dementia, age $>$ 75 years, and absence of analgesia in the ED were associated with acute in-hospital delirium.

Why does this study matter to clinicians?

Although many variables associated with delirium may not be easily modified, more careful attention to ED pain management and the provision of better analgesia are important.
\end{abstract}

\section{ABSTRACT}

Objective: The primary objective was to identify risk factors independently associated with acute in-hospital delirium within 72 hours of emergency department (ED) arrival for patients diagnosed with a hip fracture.

Methods: This was a retrospective chart review of patients ages 65 years and older presenting to one of two academic EDs with a discharge diagnosis of a hip fracture from January 1, 2014, to December 31, 2015. A multivariable logistic regression analysis was used to determine variables independently associated with the development of acute inhospital delirium within 72 hours of ED arrival.

Results: Of the 668 included patients, 181 (27.1\%) developed delirium within 72 hours of ED arrival. History of neurodegenerative disease or dementia (odds ratio [OR]: 5.7, 95\% confidence interval [Cl]: 3.9, 8.4), age > 75 (OR: $2.8,95 \% \mathrm{Cl}$ : $1.4,5.6$ ), and absence of analgesia (no opioid or nerve block) in the ED (OR: $2.1,95 \% \mathrm{Cl}: 1.3,3.2$ ) were independently associated with the development of acute in-hospital delirium; 525 (78.6\%) patients received opioid analgesia in the ED. The most common analgesics used in the ED were intravenous (IV) morphine (35.8\%), IV hydromorphone $(35.2 \%)$, or dual therapy with both IV hydromorphone and IV morphine (2.2\%). Femoral nerve blocks were initiated for $36(5.4 \%)$ patients and successfully completed in $35(5.2 \%)$ patients in the ED.

Conclusions: Advanced age and signs of dementia or neurodegenerative disease are predictors of 72-hour delirium that can be screened for during triage. Improved pain control in the ED may reduce the risk of acute in-hospital delirium.

\section{RÉSUMÉ}

Objectif: L'étude avait pour objectif principal de cerner des facteurs de risque indépendants d'apparition d'un état confusionnel aigu à I'hôpital, associés à une fracture de la hanche, dans les 72 heures suivant l'arrivée au service des urgences (SU).

Méthode: II s'agit d'un examen rétrospectif de dossiers de patients âgés de 65 ans et plus, et traités dans I'un des SU universitaires participants, pour une fracture de la hanche avérée, du $1^{\mathrm{er}}$ janvier 2014 au 31 décembre 2015. Une analyse de régression logistique plurifactorielle a servi à déterminer des variables indépendantes associées à l'apparition d'un état confusionnel aigu à I'hôpital, dans les 72 heures suivant I'arrivée au SU.

Résultats: Sur 668 patients sélectionnés, un état confusionnel aigu s'est développé dans les 72 heures suivant l'arrivée au SU, chez 181 d'entre eux (27,1\%). Des antécédents de maladie neurodégénérative ou de démence (risque relatif approché [RRA] : 5,7; IC à $95 \%: 3,9-8,4)$, un âge $>75$ ans (RRA : 2,8; IC à $95 \%: 1,4-5,6$ ) et l'absence d'analgésie (pas d'opioïdes ou de bloc nerveux) au SU (RRA : 2,1; IC à $95 \%$ : $1,3-3,2)$ se sont révélés des facteurs de risque indépendants d'état confusionnel aigu à l'hôpital. Ainsi, 525 patients (78,6 \%) ont reçu des opioïdes au SU. Les analgésiques les

From the ${ }^{*}$ Schwartz/Reisman Emergency Medicine Institute, Toronto, ON; †Sinai Health System, Toronto, ON; $¥$ Department of Family and Community Medicine, University of Toronto, Toronto, ON; and §North York General Hospital, Toronto, ON.

Correspondence to: Dr. Shelley McLeod, Department of Family and Community Medicine, University of Toronto, 206-600 University Avenue, Toronto, ON M5G 1X5; Email: shelley.mcleod@sinaihealthsystem.ca 
plus utilisés au SU étaient la morphine par voie intraveineuse (i.v.) (35,8\%), l'hydromorphone i.v. $(35,2 \%)$ ou la bithérapie composée d'hydromorphone i.v. et de morphine i.v. (2,2\%). Une anesthésie par bloc fémoral a été pratiquée chez 36 patients $(5,4 \%)$ et réussie chez $35(5,2 \%)$ d'entre eux, au SU.

Conclusion: Un âge avancé et des signes de démence ou de maladie neurodégénérative sont des facteurs prévisionnels d'apparition, dans les 72 heures suivant l'arrivée à I'hôpital, d'un état confusionnel aigu, pouvant être dépistés au moment du triage. Par ailleurs, une amélioration du soulagement de la douleur au SU peut diminuer le risque d'apparition d'un état confusionnel aigu à I'hôpital.

Keywords: analgesia, delirium, emergency department, hip fracture

\section{INTRODUCTION}

More than 35,000 Canadians experience a hip fracture each year, of whom the majority are over the age of 65 years living with osteoporosis and various other comorbidities. ${ }^{1}$ For these individuals, a hip fracture can be a catastrophic event that precipitates a steep decline in health and independence. Delirium, or acute confusion, occurs in up to $62 \%$ of patients who have experienced a hip fracture. ${ }^{2}$ Patients with delirium are significantly more likely to have a longer hospital length of stay and an increased risk of falls, pressure sores, nursing home admission, and unexpected death. ${ }^{1-3}$

Previous studies have attempted to identify risk factors associated with postoperative delirium in elderly patients. ${ }^{4-8}$ Cognitive impairment, history of dementia, advanced age, multiple comorbidities, malnutrition, baseline functional dependence, living in an institution or nursing home, visual impairment, hearing deficiency, functional impairment and psychoactive drug use, such as narcotics, benzodiazepines, and medications with anticholinergic properties, have all been associated with the development of postoperative delirium. ${ }^{4,5,9,10}$ However, these studies did not specifically address factors related to emergency department (ED) management and did not exclusively include hip fracture patients.

Untreated pain has also been associated with delirium in older adults undergoing elective surgery. ${ }^{6,11}$ Current methods of providing analgesia include acetaminophen, nonsteroidal anti-inflammatory drugs (NSAIDS), oral or parenteral opioids, and regional anesthesia techniques. ${ }^{12}$ Monotherapy with acetaminophen alone is often insufficient for the majority of hip fracture patients, and NSAIDs are largely contraindicated in this elderly patient group due to concerns regarding side effects. Opioids provide reasonable analgesia at rest but are relatively ineffective for dynamic pain, and side effects, such as nausea, vomiting, constipation, and over sedation, are common. Regional anesthesia offers an attractive alternative to systemic opioids for hip fracture patients, but femoral nerve blocks are infrequently used by emergency physicians due to the need for specialized equipment and training and the time required to perform this procedure in a busy ED setting. ${ }^{13}$

The primary objective of this study was to identify risk factors independently associated with acute inhospital delirium within 72 hours of ED arrival for patients diagnosed with a hip fracture. A secondary objective was to describe the ED pain management of patients with a hip fracture.

\section{METHODS}

\section{Study design, setting, and population}

This was a retrospective chart review of patients ages 65 years and older presenting to one of two academic EDs (an academic teaching ED with an annual census of 60,000 and a university-affiliated community hospital ED with an annual census of 100,000) in Toronto, Ontario, with an ED diagnosis of a hip fracture (intertrochanteric fracture, cervicotrochanteric fracture, subtrochanteric fracture, or other/unspecific fracture of the femoral neck/head; ICD-10 codes S72.0, S72.1, S72.2, S72.8, and S72.9. ) over a 2-year study period (January 1, 2014, to December 31, 2015). Patients were excluded if they were under the age of 65 years, did not undergo surgery for a hip fracture, had a missing ED record, were falsely labelled as having an acute hip fracture, if they were a patient in palliative care, or if they left the ED against medical advice. We excluded patients who did not undergo surgery for hip fracture repair because we had no way of following/contacting them to determine the primary outcome of delirium within 72 hours if they were not admitted to the hospital. The study protocol was approved by the Research Ethics Board at both institutions. 


\section{Study protocol}

Using a computerized, structured, data abstraction form, trained research personnel reviewed the medical records and extracted patient demographics such as age, gender, and comorbidities. Comorbidities were categorized as cardiovascular (e.g., congestive heart failure, atrial fibrillation, history of stroke), hypertension, bone health (e.g., osteoporosis and osteoarthritis), diabetes, hormonal (e.g., hypothyroidism, hyperthyroidism, and Cushing disease), mental health (e.g., depression and schizophrenia) substance use disorder, and neurodegenerative (e.g., Parkinson disease and Alzheimer disease) or dementia. Triage vital signs (i.e., heart rate, systolic blood pressure, respiratory rate, oxygen saturation, temperature, and pain score) and the Canadian Triage Acuity Scale (CTAS) score were also recorded. If a variable was not recorded in the chart, this was coded as absent. Any analgesic and anti-nausea medications administered in the ED and regional anesthesia (femoral nerve block) performed in the ED and in-hospital were recorded. Additionally, the anesthetic used for surgery and any complications occurring during surgery or in-hospital were recorded, along with total hospital length of stay. Prior to data extraction, all study variables were defined by the research team. Periodic meetings with chart abstractors and study team investigators were held to resolve disputes and monitor performance. Fifty charts were independently reviewed by two study investigators to ensure consistency in the delirium assessment.

\section{Outcomes}

The primary outcome of delirium within was defined as a positive Confusion Assessment Method (CAM) score $^{14}$ - a tool used to assess acute confusion or delirium, or a documented change from baseline cognitive status in any of the ED physician, nursing, or inpatient nursing notes through the mention of "delirious," "altered level of consciousness," "agitated," or "acute confusion." This was consistent with the definition used by Morrison et al., and attempts to account for the vagueness of delirium reporting. ${ }^{6} \mathrm{We}$ chose to use documented delirium within 72 hours of ED arrival to better capture incidences that could have been affected or influenced by the patients' ED management. We chose a 72-hour time period for several practical reasons. We wanted to ensure that there was reasonable proximity to ED arrival, but also allow adequate time to ensure that all patients had their surgery and to account for anesthetic effects to wear off prior to the determination of our primary outcome. A secondary objective was to describe the ED pain management of patients with a hip fracture.

\section{Sample size}

To estimate the sample size required for the multivariable regression model, the formula by Peduzzi et al. (1996) was used, $N=10 k / p$, where $p$ is the estimated proportion of patients who will develop delirium within 72 hours, and $k$ is the number of covariates (independent variables) to be included in the model. ${ }^{15}$ Based on a priori knowledge of delirium risk factors, it was unlikely that more than 12 covariates would be included in the final model. We estimated that $25 \%$ of ED patients with a hip fracture would experience delirium within 72 hours of ED arrival, consistent with previous studies, ${ }^{6,8,16}$ resulting in an estimated sample size of 480 patients.

\section{Data analysis}

Baseline variables of the study cohort were described using descriptive statistics and $95 \%$ confidence intervals (CIs) using standard equations. Inter-rater agreement of delirium was estimated using Cohen's kappa (k) statistic for the first 50 charts reviewed, with $\mathrm{k}<0.20$ interpreted as "poor agreement"; $\mathrm{k}=0.21$ to 0.40 interpreted as "fair agreement"; 0.41 to 0.60 interpreted as "moderate agreement"; $\mathrm{k}=0.61$ to 0.80 interpreted as "good agreement"; and k > 0.80 interpreted as "very good agreement."

Data elements were chosen with the intent of evaluating variables for model inclusion based on what is known about the epidemiology of the disease process as well as hypothesized relationships between potential independent variables and delirium. Univariable analyses were used to determine the strength of association between each variable and the primary outcome. Variables found to be independently associated with delirium $(p<0.1)$ were used as initial input into a multivariable model. A variable representing hospital site was included in the multivariable model. Backwards, stepwise multivariable logistic regression (Wald removal criterion of 0.1 ) was used to determine 
predictor variables independently associated with delirium. Likelihood ratio tests were used to determine appropriate inclusion of variables in the multivariable model. The Hosmer-Lemeshow goodness-of-fit statistic was examined to determine how well the final model described the response variable. Model discrimination was assessed by examining the area under the receiver operating characteristic curve. All data analyses were performed using Stata 13 (StataCorp. 2013. Stata Statistical Software: Release 13. College Station, TX: StataCorp LP).

\section{RESULTS}

During the study period, there were 750 ED patients with a hip fracture between the two sites. Eighty-two patients were excluded, leaving 668 patients included in the analyses (Figure 1). Patient demographics are presented in Table 1. Of the total number of patients, 181 (27.1\%) patients diagnosed with a hip fracture from the ED developed delirium within 72-hours of ED arrival. The inter-rater agreement of delirium for the first 50 charts reviewed was 1.0, indicating perfect agreement.

Median (interquartile range [IQR]) age of all included patients was $85(79,90)$ years. Median ED length of stay was similar between patients with and without delirium ( 4.8 v. 4.4 hours; $\Delta 0.4 ; 95 \%$ CI: -0.1 to 0.9 hours). Median time from triage to the operating room was also similar between groups (26.6 hours v. 26.7 hours; $\Delta$ 0.1; $95 \%$ CI: -2.3 to 2.5 hours). Patients who developed delirium had a longer hospital length of

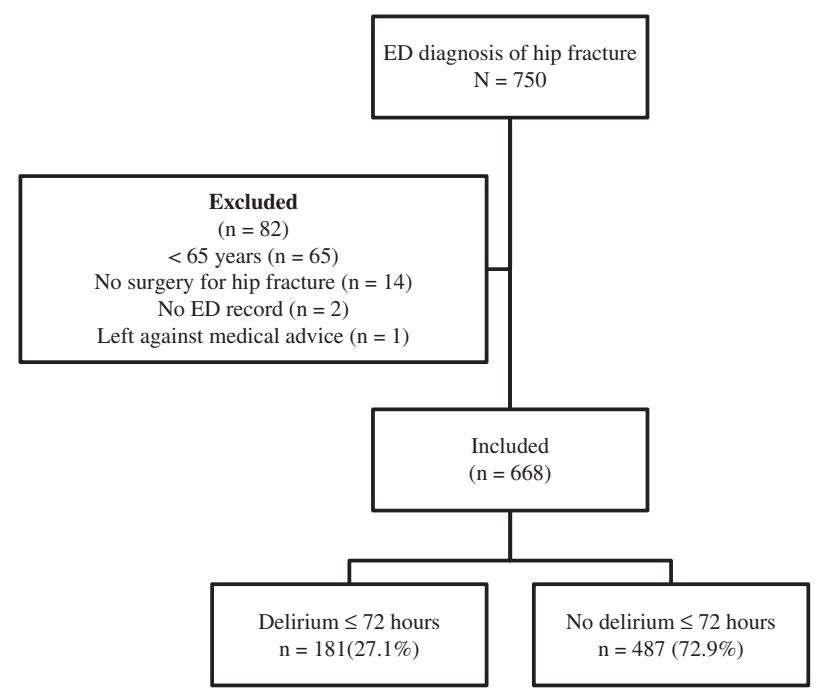

Figure 1. Flow diagram of included patients. stay compared with patients who did not develop delirium (12.8 v. 10.1 days; $\Delta$ 2.7; 95\% CI: 1.9 to 4.5 days). Overall, 138 (20.6\%) hip fracture patients did not receive either opioid analgesic or regional anesthesia while in the ED.

Results of the univariable model showing variables associated with delirium within 72-hours from ED arrival for patients presenting to the ED with a hip fracture can be viewed in Table 2. History of neurodegenerative disease or dementia (odds ratio [OR]: 5.7, 95\% CI: 3.9 to 8.4 ), age $>75$ years (OR: $2.8,95 \% \mathrm{CI}$ : 1.4 to 5.6), and absence of analgesia (no opioid or nerve block) in the ED (OR: 2.1, 95\% CI: 1.3 to 3.2 ) were independently associated with acute in-hospital delirium in the multivariable logistic regression model (Table 3). The model achieved good discrimination (area under the receiver-operating characteristic curve $=0.76$ ), and the Hosmer-Lemeshow goodness-of-fit chi-squared value was $3.77(p=0.44)$, indicating that the model was well fit to the data.

The majority of patents $(78.5 \%)$ received opioid analgesia while in the ED; the most common prescribed were hydromorphone and morphine. Patients who developed delirium with 72 hours of ED arrival were less likely to receive any pain management in the ED (68.5\% receiving analgesia v. $83.6 \%$ in the no-delirium group; $\Delta 15.1 \%$; $95 \%$ CI: $7.8 \%$ to $22.8 \%)$. Patients who received $\geq 1.5 \mathrm{mg}$ of morphine equivalents of analgesics per hour in the ED were significantly less likely to develop delirium within 72 hours of ED arrival. Femoral nerve blocks were rarely performed by ED physicians $(35 / 668 ; 5.2 \%)$. The complete summary of pain management for patients with and without delirium is presented in Table 4.

\section{DISCUSSION}

The results of this study suggest that hip fracture patients older than 75 years and those with a history of neurodegenerative disease or dementia are at increased risk for the development of delirium within 72 hours of ED arrival. Our findings also suggest that patients given no analgesia (no opioid or nerve block) in the ED are at increased risk of in-hospital delirium. Patients who developed delirium had a longer hospital length of stay compared with patients who did not develop delirium (12.8 v. 10.1 days; $\Delta$ 2.7; 95\% CI: 1.9 to 4.5 days). These patients should be viewed with increased caution in the ED, with extra care taken to screen for delirium 


\begin{tabular}{|c|c|c|c|}
\hline & $\begin{array}{c}\text { Delirium } \leq 72 \mathrm{hr} \\
\quad(\mathrm{n}=181)\end{array}$ & No delirium $\leq 72 \mathrm{hr}(\mathrm{n}=487)$ & $\begin{array}{c}\text { Total } \\
(\mathrm{N}=668)\end{array}$ \\
\hline Age $>75$ years $(\%)^{*}$ & 170 (93.9) & $386(79.3)$ & $556(83.2)$ \\
\hline Female (\%) & $135(74.6)$ & $366(75.2)$ & $501(75.0)$ \\
\hline Arrival by ambulance $(\%)^{*}$ & $168(92.8)$ & $425(87.6)$ & $593(88.8)$ \\
\hline Median (IQR) ED length of stay (hours)* & $4.8(3.4,7.2)$ & $4.4(3.1,6.2)$ & $4.5(3.2,6.5)$ \\
\hline Median (IQR) time from triage to OR (hours)* & $26.6(19.2,41.6)$ & $26.7(18.7,41.0)$ & $26.7(18.7,41.2)$ \\
\hline \multicolumn{4}{|l|}{ ED arrival time } \\
\hline Night 2400-0759 hr (\%) & $26(14.4)$ & $73(15.0)$ & $99(14.8)$ \\
\hline Day 0800-1559 hr (\%) & $79(43.9)$ & $217(44.6)$ & $296(44.4)$ \\
\hline Evening $1600-2359$ hr (\%) & $75(41.7)$ & $197(40.4)$ & $272(40.8)$ \\
\hline \multicolumn{4}{|l|}{ Triage vital signs } \\
\hline Abnormal SBP at triage (\%) & $92(50.8)$ & $259(53.2)$ & $351(52.5)$ \\
\hline Abnormal temperature at triage $(\%)$ & $100(55.2)$ & $264(54.2)$ & $364(54.5)$ \\
\hline Abnormal heart rate at triage $(\%)$ & $27(14.9)$ & $68(14.0)$ & $95(14.2)$ \\
\hline \multicolumn{4}{|l|}{ Comorbidities } \\
\hline Cardiovascular disease (\%) & $80(44.2)$ & $218(44.8)$ & $296(44.6)$ \\
\hline Hypertension (\%) & $119(65.8)$ & $301(61.8)$ & $420(62.9)$ \\
\hline Bone health comorbidity, yes (\%) & $74(40.9)$ & $207(42.5)$ & $281(42.1)$ \\
\hline Diabetes $(\%)^{*}$ & $25(13.8)$ & $100(20.5)$ & $125(18.7)$ \\
\hline Hormonal comorbidity (\%) & $33(18.2)$ & 95 (19.5) & $128(19.2)$ \\
\hline Mental health comorbidity $(\%)^{*}$ & $48(26.5)$ & $74(15.2)$ & $122(18.3)$ \\
\hline Neurodegenerative disease or dementia $(\%)^{*}$ & $120(66.3)$ & $112(23.0)$ & $232(34.7)$ \\
\hline Substance use disorder (\%) & $3(1.7)$ & $8(1.6)$ & $11(1.7)$ \\
\hline No opioid or nerve block (\%)* & $58(32.0)$ & $80(16.4)$ & $138(20.6)$ \\
\hline Median (IQR) opioid dose per hour & $1.1(0.6,1.7)$ & $1.4(0.7,2.4)$ & $1.3(0.7,2.3)$ \\
\hline
\end{tabular}

and provide optimal pain management. Receiving opioid medications at a rate of at least $1.5 \mathrm{mg}$ of meq per hour significantly reduced the likelihood of developing delirium; however, the optimal dose of opioids for prevention of delirium is not known.

Previous studies have attempted to identify risk factors associated with patients who are at higher risk of postoperative delirium following a hip fracture repair. $^{4-8}$ A recent systematic review by Yang et al. included 24 studies with a total of 5,364 patients with a hip fracture. Reported patients with preoperative cognitive impairment ([OR] 3.21; 95\% CI: 2.26 to 4.56), advanced age (standardized mean difference $0.50 ; 95 \%$ CI: 0.33 to 0.67 ), living in an institution (OR 2.94; $95 \%$ CI: 1.65 to 5.23 ), heart failure (OR 2.46; 95\% CI: 1.72 to 3.53$)$, total hip arthroplasty (OR 2.21; 95\% CI: 1.16 to 4.22), multiple comorbidities (OR 1.37; 95\% CI: 1.12 to 1.68), and postoperative morphine usage (OR 3.01;
95\% CI: 1.30 to 6.94$)$ were more likely to sustain delirium after hip surgery. Females were less likely to develop delirium after hip surgery (OR $0.83 ; 95 \%$ CI: 0.70 to 0.98$).{ }^{5}$ The OR $(3.01 ; 95 \%$ CI: $1.30,6.94)$ reported by Yang et al. was a pooled estimate derived from postoperative studies and did not address a possible dose-response relationship between opioid dose and delirium. The results of previous studies on the opioid-delirium association are inconsistent; inadequate dosing of opioids may result in pain-related delirium, whereas excessive dosing may be as likely to result in confusion. Our study was restricted to pain management for hip fracture patients while in the ED.

Oh et al. conducted a systematic review to identify preoperative clinical risk factors associated with postoperative delirium in individuals undergoing hip fracture repair to help clinicians identify high-risk patients upon admission. After adjusting for other suspected risk 
factors in multivariable models, cognitive impairment was one of the most important preoperative risk factors for delirium after hip fracture surgery, followed by body mass index, albumin levels, and multiple comorbidities. The authors concluded that a careful preoperative assessment of cognitive function would be most important in identifying patients at the highest risk of postoperative delirium; however, further study is required to determine whether early identification of these risk factors improves patient outcomes. ${ }^{16}$

Although baseline cognitive impairment is an important risk factor for postoperative delirium, $\operatorname{cog}$ nitive assessment is not routinely conducted prior to emergency hip fracture repair. Amongst older adults in the ED, delirium typically occurs as the hypoactive variety where patients may appear depressed, sedated, or lethargic and therefore easily missed if not routinely

Table 2. Results of the univariable model showing variables associated with delirium within 72-hours from ED arrival for patients presenting to the ED with a hip fracture

\begin{tabular}{lc} 
Variable & Unadjusted OR (95\% CI) \\
\hline Age $>75$ years & $4.0(2.1$ to 7.7$)$ \\
Arrival by ambulance & $1.8(0.9$ to 3.4$)$ \\
ED length of stay (per hour) & $1.1(1.0$ to 1.1$)$ \\
Time to operating room greater than & $1.0(0.7$ to 1.3$)$ \\
$\quad 24$ hours & \\
Diabetes & $0.6(0.4$ to 1.0$)$ \\
Mental health comorbidity & $2.0(1.3$ to 3.0$)$ \\
Neurodegenerative disease or & $6.6(4.5$ to 9.6$)$ \\
$\quad$ dementia & \\
No opioid or nerve block & $2.4(1.6$ to 3.6$)$ \\
Opioid dose in the ED & \\
Zero & Reference \\
$>0$ to 1.5 meq/hour & $0.5(0.3$ to 0.8$)$ \\
$>1.5$ meq/hour & $0.2(0.2$ to 0.4$)$ \\
\hline Cl = confidence interval; ED $=$ emergency department; meq = morphine equivalents; OR \\
$=$ odds ratio.
\end{tabular}

screened for. ${ }^{5,6}$ Although there are several delirium screening tools available, they are not well known by ED clinicians; it remains unclear at which stage they should be administered, and delirium screening is perceived to be too time-consuming to be performed in the ED. ${ }^{10}$ However, many studies, including those in the systematic review by $\mathrm{Oh}$ et al., demonstrate the feasibility of preoperative cognitive testing in the ED setting. ${ }^{16}$

It has been suggested that cognitive testing should become part of the standard of care for preoperative assessment for hip fracture surgery and should be screened for during the patient's initial ED assessment. It has been recommended that delirium screening occurs at least once every 12 hours while the patient is in hospital, after transitions between in-patient settings, and upon any change in medical status. ${ }^{17}$ However, in our study, only one site used the CAM score to screen for acute confusion at triage, with only $28.4 \%$ of patients having a completed CAM score recorded, suggesting future efforts should focus on initiatives to improve delirium screening compliance by ED personnel. Early identification of confusion through routine delirium screening would allow for hospital-based delirium prevention approaches, which could be implemented in the ED. Examples of such strategies include orienting the patient to person, place, and time (involving family and caregivers when possible); creating an environment that provides context (a room with a clock); ensuring use of glasses/hearing aids as appropriate; and optimizing the use of drugs associated with delirium. ${ }^{4,10}$

In our study, patients who did not develop delirium received a higher dose of opioid analgesia compared with those who developed delirium. However, controlling pain is often challenging in this population due to advanced patient age, decreased cognitive function, and various comorbidities. ${ }^{3,6}$ Abou-Setta et al.

Table 3. Results of the multivariable regression model showing independent predictors of delirium within 72 hours from ED arrival for patients presenting to the ED with a hip fracture

\begin{tabular}{|c|c|c|}
\hline Variable & $\begin{array}{l}\text { Adjusted OR } \\
(95 \% \mathrm{Cl})\end{array}$ & $\begin{array}{c}\beta \text {-coefficient } \\
(95 \% \mathrm{Cl})\end{array}$ \\
\hline No ED analgesia (no nerve block or narcotic) & $2.1(1.3$ to 3.2$)$ & $0.7(0.3$ to 1.2$)$ \\
\hline Age $>75$ years & $2.8(1.4$ to 5.6$)$ & $1.0(0.4$ to 1.7$)$ \\
\hline History of neurodegenerative disease or signs of dementia & 5.7 (3.9 to 8.4) & $1.7(1.4$ to 2.1$)$ \\
\hline
\end{tabular}




\begin{tabular}{|c|c|c|c|}
\hline & Delirium $\leq 72 \mathrm{hrs}(\mathrm{n}=181)$ & No delirium $\leq 72 \mathrm{hrs}(\mathrm{n}=487)$ & Difference \\
\hline No opioid or nerve block (\%) & $57(31.5)$ & $80(16.4)$ & $15.1(7.8 \text { to } 22.8)^{*}$ \\
\hline Opioid analgesia only (\%) & $118(65.2)$ & $378(77.6)$ & $12.4(4.8 \text { to } 20.4)^{*}$ \\
\hline Femoral nerve block only (\%) & $3(1.7)$ & $3(0.6)$ & $1.0(-0.5$ to 4.2$)$ \\
\hline Opioid analgesia and femoral nerve block (\%) & $3(1.7)$ & $26(5.3)$ & $3.7(0.1 \text { to } 6.3)^{*}$ \\
\hline \multicolumn{4}{|l|}{ Analgesia Use } \\
\hline Hydromorphone, yes (\%) & $58(32.0)$ & 199 (40.9) & $8.8(0.5 \text { to } 16.6)^{*}$ \\
\hline Morphine, yes (\%) & $64(35.6)$ & $194(39.8)$ & $4.5(-0.4$ to 12.5$)$ \\
\hline Acetaminophen + oxycodone, yes (\%) & $3(1.7)$ & $15(3.1)$ & $1.4(-1.9$ to 3.6$)$ \\
\hline Acetaminophen + codeine, yes $(\%)$ & $2(1.1)$ & $22(4.5)$ & $3.4(0.2 \text { to } 5.8)^{*}$ \\
\hline Codeine, yes (\%) & $1(0.6)$ & $4(0.8)$ & $0.2(-2.3$ to 1.6$)$ \\
\hline No opioid analgesia (\%) & $60(33.2)$ & $83(17.0)$ & $16.1(8.7 \text { to } 23.9)^{*}$ \\
\hline \multicolumn{4}{|l|}{ Pain Management } \\
\hline Median (IQR) opioid dose per hour & $1.1(0.6,1.7)$ & $1.4(0.7,2.4)$ & $0.3(0.1 \text { to } 0.4)^{*}$ \\
\hline \multicolumn{4}{|l|}{ Opioid rate breakdown } \\
\hline Zero (\%) & $65(36.1)$ & $86(17.8)$ & $18.2(10.6 \text { to } 26.1)^{*}$ \\
\hline$>0$ to $1.5 \mathrm{meq} /$ hour $(\%)$ & 79 (43.9) & $209(43.4)$ & $0.4(-7.9$ to 8.9$)$ \\
\hline$>1.5$ meq/hour (\%) & $36(20.0)$ & $186(38.7)$ & $18.7(11.0 \text { to } 25.5)^{*}$ \\
\hline \multicolumn{4}{|l|}{ Analgesia Dose } \\
\hline Median (IQR) hydromorphone (meq) & $6.7(3.4,10.0)$ & $6.7(3.4,13.4)$ & $0(0$ to 2.0$)$ \\
\hline Median (IQR) morphine (meq) & $4(4,6)$ & $4(4,8)$ & $0(0$ to 0$)$ \\
\hline Median (IQR) acetaminophen + oxycodone (meq) & $0.3(0.3,0.5)$ & $0.3(0.3,0.5)$ & $0(0$ to 0.25$)$ \\
\hline Median (IQR) acetaminophen + codeine (meq) & $4.5(4.5,4.5)$ & $4.5(2.8,4.5)$ & $0(0$ to 1.5$)$ \\
\hline Median (IQR) codeine (meq) & $2.3(2.3,2.3)$ & $4.5(3.4,4.5)$ & $\mathrm{N} / \mathrm{A}^{+}$ \\
\hline
\end{tabular}

conducted a systematic review of the comparative effectiveness of pain management strategies for hip fracture and found evidence from four randomized controlled trials $(\mathrm{n}=461)$ and two cohort studies $(\mathrm{n}=$ 634) to suggest that nerve blocks are effective for relieving acute pain and reducing delirium in hip fracture patients. ${ }^{12}$ The authors also concluded that perioperative use of regional anesthesia independently reduced the severity and duration of delirium and that preoperative administration was more effective than postoperative administration. Although our study indicated that improved analgesia reduced the incidence of delirium amongst older hip fracture patients, regional anesthesia was rarely used, and we had insufficient data to determine the effectiveness of femoral nerve blocks in preventing delirium.

\section{Limitations}

This study has several limitations. Due to the retrospective nature of the study, we can only report what was documented in the patient record. The primary outcome of delirium was defined as a positive CAM score, or a documented change from baseline cognitive status in any of the ED physician, nursing, or inpatient nursing notes through the mention of "delirious," "altered level of consciousness," "agitated," or "acute confusion." Due to the retrospective nature of the study, it was difficult to differentiate between prevalent delirium (arrived with delirium) and incident delirium (developed after arriving in the ED). It may be possible that some patients experienced delirium, which was undocumented in the chart and therefore not captured in our study. Alternatively, it is possible that psychiatric symptoms might have been misclassified as "delirium" when patients were severely ill or suffering from dementia (false-positive), as has been shown by Inouye et al. ${ }^{18}$

The impact of ED management may have been confounded by inpatient variables, including the surgery; however, our interest was in establishing the impact of analgesia provided during the ED stay. We chose a 72-hour time period for several practical reasons. We wanted to ensure there was reasonable 
proximity to ED arrival, but also allow adequate time to ensure that all patients had their surgery, and to account for anesthetic effects to wear off prior to the determination of our primary outcome.

Due to the low number of regional nerve blocks performed by ED physicians, we were unable to independently investigate the association between femoral nerve blocks and incidence of delirium. Additionally, CAM scores were used at only one of the two included sites, so the incidence of delirium may not have been consistently documented between the two hospitals. It is also possible that some of the observed variation in the collected data were influenced by error or bias from the data abstractor, who was not blinded to the objective of this descriptive study. The data included in this study are from 2014 to 2015, but we do not believe that the pain management for ED hip fracture patients has changed. Finally, this was a small study, and physician practice was limited to two hospitals. Although clinical management strategies appeared similar across a large number of physicians at both institutions, our findings may not be generalizable to other settings.

\section{CONCLUSIONS}

History of neurodegenerative disease or dementia, age $>75$ years, and absence of analgesia (no opioid or nerve block) in the ED were risk factors independently associated with acute in-hospital delirium within 72 hours of ED arrival for patients diagnosed with a hip fracture. The results of this study suggest that patients at an advanced age and those with a history of neurodegenerative disease or dementia are at increased risk for confusion, which complicates and prolongs hospital length of stay. Although many variables associated with delirium may not be easily modified, more careful attention to ED pain management and the provision of better analgesia are important. Our findings indicate that appropriate pain management in the ED, through the use of opioid medications or regional anesthesia, may reduce the risk of in-hospital delirium for patients diagnosed with a hip fracture. Delirium may be a preventable condition amenable to hospital-based prevention strategies, which could be implemented in the ED.

Competing interests: None declared.

\section{REFERENCES}

1. Riddell M, Ospina M, Holroyd-Leduc JM. Use of femoral nerve blocks to manage hip fracture pain among older adults in the emergency department: a systematic review. CFEM 2016;18(4):245-52.

2. Zywiel MG, Hurley RT, Perruccio AV, et al. Health economic implications of perioperative delirium in older patients after surgery for a fragility hip fracture. 7 Bone foint Surg Am 2015;97:829-36.

3. Rosen T, Connors S, Clark S, et al. Assessment and management of delirium in older adults in the emergency department: literature review to inform development of a novel clinical protocol. Adv Emerg Nurs 7 2015;37(3):183E3.

4. Inouye SK, Viscoli CM, Horwitz RI, et al. A predictive model for delirium in hospitalized elderly medical patients based on admission characteristics. Ann Intern Med 1993;119 (6):474-81.

5. Yang $\mathrm{Y}$, Zhao X, Dong $\mathrm{T}$, et al. Risk factors for postoperative delirium following hip fracture repair in elderly patients: a systematic review and meta-analysis. Aging Clin Exp Res 2017;29:115-26.

6. Morrison RS, Magaziner J, Gilbert M, et al. Relationship between pain and opioid analgesics on the development of delirium following hip fracture. 7 Gerontol 2003;58(1):76-81.

7. Lee HB, Mears SC, Rosenberg PB, et al. Predisposing factors for post-operative delirium after hip fracture repair among patients with and without dementia. 7 Am Geriatr Soc 2011;59(12):2306-13.

8. Bruce AJ, Ritchie CW, Blizard R, et al. The incidence of delirium associated with orthopedic surgery: a metaanalytic review. Int Psychogeriatr 2007;19(2):197-214.

9. Han JH, Wilson A, Ely EW. Delirium in the older emergency department patient - a quiet epidemic. Emerg Med Clin North Am 2010;28(3):611-31.

10. Han JH, Zimmerman EE, Cutler N, et al. Delirium in older emergency department patients: recognition, risk factors, and psychomotor subtypes. Acad Emerg Med 2009;16(3):193200.

11. Morrison RS, Magaziner J, Koval KJ, et al. The impact of post-operative pain on outcomes following hip fracture. Pain 2003;103:303-11.

12. Abou-Setta AM, Beaupre LA, Rashiq S, et al. Comparative effectiveness of pain management interventions for hip fracture: a systematic review. Ann Intern Med 2011;155 (4):234-45.

13. Morrison RS, Dickman E, Hwang U, et al. Regional nerve blocks improve pain and functional outcomes in hip fracture: a randomized controlled trial. 7 Am Geriatr Soc 2016;64 (12):2433-9, doi: doi:10.1111/jgs.14386.

14. Inouye SK, van Dyck CH, Alessi CA, et al. Clarifying confusion: the confusion assessment method. A new method for detection of delirium. Ann Intern Med 1990;113 (12):941-8.

15. Peduzzi P, Concato J, Kemper E, et al. A simulation study of the number of events per variable in logistic regression analysis. 7 Clin Epidemiol 1996;49(12):1373-9. 
16. Oh ES, Sieber FE, Leoutsakos J, et al. Sex differences in hip fracture surgery: preoperative risk factors for delirium and postoperative outcomes. 7 Am Geriatr Soc 2016;64:1616-21.

17. Health Quality Ontario, Ministry of Health and Long-Term Care. Care for people with fragility fractures: Health Quality Ontario; 2017. Available at: http://www.hqontario.ca/Evi
dence-to-Improve-Care/Quality-Standards/View-all-QualityStandards/Hip-Fracture (last accessed 23 March 2018).

18. Inouye SK, Leo-Summers L, Zhang Y, et al. A chart-based method for identification of delirium: validation compared with interviewer ratings using the Confusion Assessment Method. 7 Am Geriatr Soc 2005;53(2):312-8. 